\title{
Fulminant hepatic failure secondary to hydroxychloroquine
}

\author{
A J Makin, J Wendon, S Fitt, B C Portmann, Roger Williams
}

\begin{abstract}
Hydroxychloroquine is widely used in rheumatological disease but hepatic side effects have not been reported previously. Two cases are described of fulminant hepatic failure developing after the start of hydroxychloroquine treatment for a chronic rheumatological disorder. In both cases the symptoms of liver disease developed within two weeks of starting hydroxychloroquine and rapidly progressed to fulminant hepatic failure and in neither case was there any pre-existing liver disease. One patient had emergency orthotopic liver transplantation and the other died before a donor organ became available.

(Gut 1994; 35: 569-570)
\end{abstract}

Adverse drug reactions are a significant cause of massive hepatic necrosis accounting for 7 to $15 \%^{12}$ of reported cases of fulminant hepatic failure. About 600 drugs have been suspected of possible hepatotoxicity ${ }^{3}$ but in most cases the responsible drugs are isoniazid, anti-depressants, non-steroidal anti-inflammatory drugs, anti-convulsants, halothane and its derivatives. Hydrozychloroquine, a $\beta$-hydroxylated $N$-ethyl substituent of chloroquine, is a 4-aminoquinolone widely prescribed in rheumatological disease. Despite its wide use only one case of hepatitis and one case of jaundice have been notified to The Committee on Safety of Medicines since 1963 and fulminant hepatic failure has never been described as a side effect. Severe side effects of the drug including retinopathy and marrow suppression usually only occur when the recommended dose is exceeded. Fulminant hepatic failure has, however, been reported in association with other 4-aminoquinolones, amodiaquine ${ }^{4}$ and mepacrine, ${ }^{3}$ and with quinine, ${ }^{3}$ a closely related compound. All of these agents are often prescribed as anti-malarial drugs. In this report we describe two cases of fulminant hepatic failure associated with hydroxychloroquine treatment.

The Institute of Liver Studies, King's College Hospital and School of Dentistry, London A J Makin

J Wendon

$S$ Fitt

B C Portmann

R Williams

Correspondence to:

Dr Roger Williams, Institute Dr Roger Williams, Institu
of Liver Studies, King's of Llver Studies, King's College Hospital, Dens
Hill, London SE5 9RS Hill, London SES 9 RS. 10 August 1993

\section{Case 1}

A 27 year old women was admitted with a two week history of nausea and vomiting and four days of increasing jaundice. Two weeks before the onset of these symptoms she had been prescribed hydroxychloroquine $200 \mathrm{mg}$ twice daily for severe systemic lupus erythematosus from which she had suffered for 12 years. Her systemic lupus erythermatosus affected only the joints and she had been treated long term with steroids (30 mg prednisolone at time of admission) and for the previous year with ibuprofen $400 \mathrm{mg}$ twice daily. Her liver function tests had always been normal before this presentation. On admission she had jaundice, there were no stigmata of chronic liver disease, bilirubin was $161 \mu \mathrm{mol} / \mathrm{l}$, alanine aminotransferase $2575 \mathrm{U} / \mathrm{l}$, international normalised ratio (INR) $3 \cdot 3$. Hepatitis serology for $A, B$, and C were negative and an abdominal ultrasound showed no abnormality with a normal sized liver and spleen. Over the next 48 hours she deteriorated, with deepening encephalopathy and the development of cerebral oedema. Bilirubin and INR increased to $302 \mu \mathrm{mol} / \mathrm{l}$ and 10.6 respectively. At this point she was listed for urgent liver transplantation, but her condition deteriorated further with the development of sepsis and hypotension and she died four days after admission before a graft became available. Histological examination of the liver showed submassive necrosis of the parenchyma with near complete hepatocyte drop out and only a few duct like structures identifiable in the periportal areas. There was a moderate inflammatory cell infiltrate throughout the liver, which included pigmented macrophages and eosinophils. The infiltrate was heaviest in the portal areas where there were greater numbers of lymphocytes and plasma cells. The findings were consistent with a drug related cause.

\section{Case 2}

A 16 year old girl was admitted with a short history of deepening jaundice and general malaise. Juvenile Still's disease had been diagnosed two years previously and she had been treated since then with indomethacin $50 \mathrm{mg}$ three times a day. Hydroxychloroquine $200 \mathrm{mg}$ twice daily had been started two weeks before this episode when her arthritis had flared up. At the time of admission she was deeply jaundiced but with no organomegaly or stigmata of chronic liver disease. Initial investigation showed a prothrombin time of 50 seconds (control 15 seconds), bilirubin $417 \mu \mathrm{mol} / \mathrm{l}$, aspartate aminotransferase $544 \mathrm{U} / 1$, and she was in renal failure with a creatinine of $347 \mu \mathrm{mol} / \mathrm{l}$. A smooth muscle antibody titre was positive 1 in 120 and IgG was raised at 31.5 but serology for hepatitis $A$ and $B$ were negative. Her condition deteriorated rapidly with the development of grade IV encephalopathy with cerebral oedema and her prothrombin time increased to more than 150 seconds. She had urgent liver transplant three days after admission but died six days after transplant with multiple organ failure. Histological examination of the resected liver showed massive necrosis of the parenchyma with confluent multi-acinar cell drop out. There was a moderately heavy inflammatory infiltrate throughout with pigment ladened macrophages, 
eosinophils, and a considerably increased plasma cell count. There was no evidence of chronic liver disease and the findings were consistent with a drug related or viral cause.

\section{Discussion}

Both patients developed fulminant hepatic failure resulting from massive liver cell necrosis, which can reasonably be attributed to hydroxychloroquine for the following reasons. Firstly, there was no serological evidence to suggest a recently acquired hepatitis $A, B$, or $C$ infection and there was no risk factors to suggest a non- $A$, non-B viral hepatitis. Secondly, there were no clinical findings or histological changes to suggest chronic liver disease and neither could alcohol be implicated. Thirdly, fulminant hepatic failure developed two weeks after hydroxychloroquine treatment had started. Finally, both cases had longterm treatment with both non-steroidal anti-inflammatory drugs (NSAIDs) and corticosteroids and no other new treatment had been given. There was no history of any other drug ingestion.

Although it is reported that abnormal liver function tests do occur in up to $25 \%$ of cases of systemic lupus erythermatosus, in only $8 \%$ can they be attributed to the disease itself. ${ }^{5}$ Hepatic failure has also been described in systemic lupus erythermatosus but in all the reported cases chronic liver disease was established histologically. ${ }^{6}$ The two cases reported here have histological changes in the liver of an acute massive necrosis with no evidence of chronic disease, entirely consistent with drug related hepatotoxicity.
NSAIDs are a recognised cause of abnormal liver function tests and fulminant hepatic failure ${ }^{3}$ but the overall incidence of hepatotoxicity is low and results from an idiosyncratic reaction to the drug. This is confirmed in a recent study that suggests that the incidence of acute liver injury in patients on longterm treatment with NSAIDs is not significantly greater than in subjects who have never used them. ${ }^{?}$

The onset of fulminant hepatic failure in these patients so soon after the start of hydroxychloroquine treatment, with histological changes compatible with a drug related cause, implicates this agent as the probable cause. This association is supported further by the fact that although hydroxychloroquine itself has never been reported to cause fulminant hepatic failure, other 4-aminoquinolones have been. This suggests that all members of this group of drugs should be considered as potentially hepatotoxic.

1 Williams R, Wendon J. Clinical syndrome and aetiology of fulminant hepatic failure. Proceedings of Eleventh BSG/ Smith Kline and French International Workshop 1990: 1-5. 2 Bernau J, Reuff B, Benhamou J-P. Fulminant and subfulminant liver failure: definitions and causes. Semin Liver Dis 1986; 6: 97-106.

3 Pessayre D, Larrey D. Acute and chronic drug-induced hepatitis. Baillières Clin Gastroenterol 1988; 2: 385-422.

4 Bernau J, Larrey D, Campillo B, Degott C, Verdier F, Rueff B et al. Amodiaquine-induced fulminant hepatitis. $\mathcal{F}$ Hepato 1988; 6: 109-12.

5 Miller MH, Urowitz MB, Gladman DD, Blendis LM. The live in systemic lupus erythematosus. $Q \mathcal{F}$ Med 1984; 211 : 401-9.

6 Runyon BA, LaBrecque DR, Anuras S. The spectrum of liver disease in systemic lupus erythemataosus. Am $\mathcal{F}$ Med 1980; 69: 187-94.

7 Rodriguez LAG, Gutthann SP, Walker AM, Lueck L. The role of non-steroidal anti-inflammatory drugs in acute liver injury. $B M \mathcal{F}$ 1992; 305: 865-8. 